\title{
Wave Propagation in an Optimized Tunable Multi- channel Flter based on One-dimensional Nano Superconductor Photonic Crystal
}

\author{
Ali Baseri \\ Shiraz University of Technology \\ Alireza Keshavarz ( $\nabla$ keshavarz@sutech.ac.ir) \\ Shiraz University of Technology https://orcid.org/0000-0003-3637-7005
}

\section{Research Article}

Keywords: Low-temperature superconductor , Photonic band-gap , Terahertz multi-channel filter , Transfer matrix method

Posted Date: April 20th, 2021

DOI: https://doi.org/10.21203/rs.3.rs-423259/v1

License: (c) (1) This work is licensed under a Creative Commons Attribution 4.0 International License.

Read Full License 


\title{
Wave propagation in an optimized tunable multi-channel filter based on one-dimensional nano superconductor photonic crystal
}

\author{
Ali Baseri • Alireza Keshavarz
}

Received: date / Accepted: date

\begin{abstract}
Although there has been a focus on $\mathrm{THz}$ filters so far, there is a significant deficiency in advancing low-temperature $\mathrm{THz}$ filters. According to the needs, we proposed a tunable $\mathrm{THz}$ filter that selectively permits the desired incident frequencies to be propagated in relevance with our purpose. The presence of a low-temperature nano superconductor and an undoped semiconductor layers in the proposed structure resulted in a multi-channel $\mathrm{THz}$ filter, which could be highly tuned with several parameters such as lattice constants, applied temperature, etc. The achieved transmittance spectra revealed that the emerged transmittance couples and stacks follow exact regulations. Furthermore, the structure exhibited omnidirectional band-gaps for both TE and TM polarized waves. Moreover, the use of a central defect layer gave some transmittance defect modes in the forbidden areas. This structure could be used in some $\mathrm{THz}$ devices such as switches, optimized sensors as well as in space industry and telecommunications.
\end{abstract}

Keywords Low-temperature superconductor · Photonic band-gap · Terahertz multi-channel filter · Transfer matrix method

\section{Introduction}

Terahertz $(\mathrm{THz})$ spectrum is possibly the latest spectrum, that has been added to the electromagnetic (EM) spectra [1]. The energy range of the spectrum puts wave-matter interaction at the "bridge" between the quantum interpretation of interactions at visible light and higher energies (mostly particle-like behavior of light), and the continuum form of interpretation of interactions at lower energies (mostly wave-like behavior of light) [2]. The technology that arises from the spectrum is called THz technology. In 2004, Technology Review's editors selected

\section{A. Baseri}

Physics Department, Shiraz University of Technology, Shiraz, Iran

E-mail: a.baseri@sutech.ac.ir

A. Keshavarz

Physics Department, Shiraz University of Technology, Shiraz, Iran

E-mail: keshavarz@sutech.ac.ir 
$\mathrm{THz}$ technology as one of "10 emerging technologies that will change your world" [3]. $\mathrm{THz}$ technology is used in the following fields: medicine [4,5], biology [6], security [7], non-destructive evaluation [8], agriculture [9], telecommunication [10], pharmaceutical [11], spectroscopy [12,13], cultural heritage [14], etc. The abovementioned applications show that the $\mathrm{THz}$ technology is an interdisciplinary technology. Using photonic crystal (PC) structures is the easiest and most efficient way of constructing filters.

Over recent decades, PCs, that are periodically constituted from two or more media, have been under a lot of focus due to their photonic band-gaps (PBGs), which make EM wave propagation to be forbidden at some frequency ranges. Controlling, manipulating and amplifying EM waves are the most noteworthy and intriguing consequences, which PCs could demonstrate from themselves. The PBG based PCs are typically used in the following fields: microfluidic and biomedical sensors [15], polarization splitter [16], solar cells [17,18], Terahertz Sensor [19,20], integrated circuits [21], photovoltaic devices [22], photonic filters [23], etc.

Superconductors have also been considered in various fields of study due to their unique properties. Since the permittivity of a superconducting material can be tuned with temperature and external magnetic field, superconducting PC has an advantage over metallic and dielectric PCs [24].

Given that propositions of the functional $\mathrm{THz}$ devices are growing up, the demands for developing the tunable $\mathrm{THz}$ filters increase for the narrow- and broadband $\mathrm{THz}$ systems, and $\mathrm{THz}$ circuits. The realization of developments in $\mathrm{THz}$ filters paves the way for the filters and $\mathrm{THz}$ devices to be advanced in other photonic systems, and to reach high quality in $\mathrm{THz}$ systems. In particular, $\mathrm{THz}$ PBG-based filters, in which they operate based on the interference phenomenon, have attracted a huge number of researches. Narrow-band transmission THz filters are a type of filters, which offer the thin transmission peaks as an outcome. These types of filters could be achieved by utilizing a defect layer in the structure of the PC. In fact, the narrow-band transmission mode is the consequence of the confinement effect of the photonic barriers. The band passes are originated from constructive interference of the incidence beam, which is reflected and transmitted from the interface of the layers within the structure, while the stop-bands are originated from destructive interference. To achieve any types of the interferences at desired frequency, the wave dispersion of the incident beam needs to be adjusted [25]. Moreover, the material and the arrangement of the constituent layers play a main role in realizing the desirable interference. The filters, whose transmittance spectra are comprised of several narrow band passes are called multi-channel filters [26]. This type of filter is usually constructed with the deposition of a single or several defect layers of quantum well structures within the PC arrangements.

Although, the emergent modern THz technology almost goes back to the 1970s, investigations of THz spectrum on PCs date back to the 1990s. Padilla et al. [27] proposed a high-pass microstructure filter, which contained metamaterial. The structure is the first 2D PC-based THz filter. Drysdale et al. [28] designed a metallic $\mathrm{THz}$ PC filter, which could be tuned, mechanically. Then, Němec et al. [29] composed a smart narrow-band transmission filter contained a $\mathrm{KTaO}_{3}$ defect layer, whose relative tunability reached up to $20 \%$. Subsequently, a ferroelectric defect layer of $\mathrm{SrTiO}_{2}$ was deposited in an alternating structure of quartz and highpermittivity ceramic layers, thus the tunability of the structure reached up to $60 \%$. Hung et al. [30] declared that the thermal tunability of the transmission band-gaps 
and defect modes could be realized by the deposition of an InSb semiconductor defect layer on the 1DPC structure as THz filter. The above-mentioned studies are almost the basis of formation of $\mathrm{THz}$ PC filters, therefore many investigations have been done, afterward. Srivastava [31] studied defect mode of the transmittance spectrum relevant to a 1DPC consisting of a high- and low-temperature superconductor. Aly and Sabra [32] theoretically reported a 1D metamaterial PC consisted of high-temperature superconductor and semiconductor constituents, which interacted with near-infrared waves. The structure filtered some parts of the frequency range of $0 \mathrm{GHz}$ to $2.2 \mathrm{GHz}$. Chaves and Posada [33] investigated the transmittance spectrum of a 1DPC, which was fabricated from the semiconductor GaAs and the high-temperature superconductor $\mathrm{HgBa}_{2} \mathrm{Ca}_{2} \mathrm{Cu}_{3} \mathrm{O}_{8+\delta}$, under several parameter changes such as hydrostatic pressure, angle of incidence, and temperature.

Since using the unconventional substances overcomes drawbacks of PCs, the following substances with their own special effects and properties have been used in them: superconductors [34], metamaterials [35], graphene [36], liquid crystal [37], $\mathrm{VO}_{2}$ [38], etc. Quasi-periodic (QP) and mixed-quasi-periodic (MQP) [23] arrangements could also be useful options for the configuration of the structure to achieve the desirable outcomes in accordance with the desired applications.

In this study, a special type of 1DPC-based THz filter containing two different types of constituent layers as semiconductor ( $I n S b)$ layer and low-temperature superconductor $(\mathrm{Nb})$ nano defect layer is proposed. In the current simulation, the transmission spectrum of the proposed filter is investigated via the Drude model [39] and the two-fluid theory [40] for several parameters such as temperature, number of periods of the structure, lattice constants, incidence angle of the incident beam, etc.

In this article, theoretical models and the basic equations relevant to the utilized superconductor and semiconductor are presented in section 2. The proposed structure, numerical results, which use the transfer matrix method (TMM), and discussion are presented in section 3. Finally, the conclusion is provided in section 4.

\section{Theoretical models and basic equations}

The proposed structure consists of InSb semiconductor $(A)$ layer and a lowtemperature superconductor $(B)$ as defect layer with the thicknesses of $d_{A}$ and $d_{B}$, respectively. Therefore, the arrangement of the structure could be shown as $(A B)^{M}$, where $M$ is the period number. According to the Drude model, the relative permittivity of $I n S b$ in $\mathrm{THz}$ range is defined as [27]:

$$
\varepsilon_{A}(\omega)=\varepsilon_{\infty}-\frac{\omega_{P}^{2}}{\omega^{2}-i \gamma_{s e m} \omega}
$$

where $\varepsilon_{\infty}$ is the static permittivity of $I n S b ; \omega$ expresses the angular frequency, and $\gamma_{\text {sem }}$ shows the loss of EM waves in the semiconductor. $\omega_{P}$ indicates the plasma frequency, which is defined as $\sqrt{N e^{2} / \varepsilon_{0} m^{\star}}$. Here, $N$ is intrinsic carrier density; $m^{\star}$ is the effective mass of free carrier; $e$ represents the electron charge, and $\varepsilon_{0}$ is the free space dielectric constant. The intrinsic carrier density $N$ of $\operatorname{InSb}$, in $m^{-3}$, can be given as:

$$
N=5.76 \times 10^{20} T^{3 / 2} \exp \left(\frac{-0.13}{k_{B} T}\right)
$$


Here, temperature $T$ is in Kelvin, and $k_{B}$ is the Boltzman constant. The exponential coefficient on the relation reveals that the plasma frequency highly depends on the temperature. Correspondingly, the tunability of the permittivity $\varepsilon_{A}(\omega)$ of InSb is proportionally temperature-dependent in the far-infrared portion of $\mathrm{THz}$ regime.

In accordance with the two-fluid theory, the relative permittivity of a lossless superconductor is given by [41]:

$$
\varepsilon_{B}(\omega)=1-\frac{c^{2}}{\omega^{2} \lambda_{0}^{2}}\left[1-\left(\frac{T}{T_{C}}\right)^{q}\right]
$$

where $c$ is the speed of light in free space; $T_{C}$ represents the critical temperature of the superconductor, and $\lambda_{0}$ indicates the London penetration depth at $T=0 \mathrm{~K}$, thus the parameter is defined as follows at the other temperatures:

$$
\lambda_{L}=\frac{\lambda_{0}}{\sqrt{1-G}} ; G=\left(\frac{T}{T_{C}}\right)^{q}
$$

where $q$ refers to the type of superconductor. Principally, $q=2$ and 4 are respectively considered for high- and low-temperature superconductors. In the current study, $q=4$ is chosen for $G$. Although, in the above relative permittivity relation the loss originated from conducting electrons is ignored, it has been properly adapted to the experimental results. Furthermore, many valuable studies have also used the approximation. On the other hand, the conductivity of a superconductor is defined as follows [32]:

$$
\sigma=\sigma_{r}-j \sigma_{i}
$$

where $\sigma_{r}$ represents the loss of the superconductor, and $\sigma_{i}$ indicates the conductivity of a lossless superconductor. Accordingly, the conductivity of our lossless superconductor is:

$$
\sigma \simeq \sigma_{i}=\frac{1}{\omega \mu_{0} \lambda_{L}^{2}}
$$

where $\mu_{0}$ is the permeability constant in free space. The conductivity at a temperature greater than $T_{C}$ is inverse of the specific resistivity in $\rho \sigma=1$ [42]. Thus, the resistivity of the superconductor could be redefined as the following relation, in accordance with relations (4) and (6):

$$
\rho=\frac{\omega \mu_{0} \lambda_{0}^{2}}{1-\left(\frac{T}{T_{C}}\right)^{q}}
$$

where its unit is expressed as $\Omega m$ in the SI system. The total relative permittivity in addition to the loss term has the following definition:

$$
\varepsilon_{B}(\omega)=1-\frac{c^{2}}{\omega^{2} \lambda_{0}^{2}}\left[1-\left(\frac{T}{T_{C}}\right)^{4}\right]-\frac{c^{2}}{\omega\left(\omega+i \gamma_{s u p}\right) \lambda_{0}^{2}}\left(\frac{T}{T_{C}}\right)^{4},
$$

where $\gamma_{\text {sup }}$ expresses damping of the normal conducting electrons.

TMM is an appropriate and exact approach for obtaining the transmittance spectrum of an incident beam of the plain EM waves, which entered a layered medium from free space or any other media, with $z$ direction at an incidence angle $\theta$. The electric and magnetic components of the incident wave in the $j$ th layer 
$(j=A, B)$ can be related to its back and forward layers via the following transfer matrix [30]:

$$
M_{j}\left(d_{j}, \omega\right)=\left[\begin{array}{cc}
\cos \left(k_{j z} d_{j}\right) & \frac{-i}{p_{j}} \sin \left(k_{j z} d_{j}\right) \\
-i p_{j} \sin \left(k_{j z} d_{j}\right) & \cos \left(k_{j z} d_{j}\right)
\end{array}\right],
$$

where $k_{j z}=(\omega / c) \sqrt{\varepsilon_{j}} \sqrt{\mu_{j}} \sqrt{1-\left(\sin ^{2} \theta / \varepsilon_{j} \mu_{j}\right)}$ is the wave vector, and $\varepsilon_{j}$ and $\mu_{j}$ are respectively the permittivity and permeability of the $j$ th layer. According to the Blochs theorem, the dispersion relation of a periodic layered medium that interacts with an incident EM wave under the boundary conditions is given by:

$$
\cos (K D)=\cos \left(k_{A z} d_{A}\right) \cos \left(k_{B z} d_{B}\right)-\frac{1}{2}\left(\frac{q_{A}}{q_{B}}+\frac{q_{B}}{q_{A}}\right) \sin \left(k_{A z} d_{A}\right) \sin \left(k_{B z} d_{B}\right)
$$

where

$$
\begin{aligned}
& p_{j s}=\left(\sqrt{\varepsilon_{j}} / \sqrt{\mu_{j}}\right) \sqrt{1-\left(\sin ^{2} \theta / \varepsilon_{j} \mu_{j}\right)}, \\
& p_{j p}=\left(\sqrt{\mu_{j}} / \sqrt{\varepsilon_{j}}\right) \sqrt{1-\left(\sin ^{2} \theta / \varepsilon_{j} \mu_{j}\right)},
\end{aligned}
$$

are respectively in relevance with TE and TM modes. Hence, the total transfer matrix of the multi-layered structure can be obtained by multiplying each single transfer matrix of the layers, as follows:

$$
M_{T}=\left(\begin{array}{ll}
M_{A} & M_{B}
\end{array}\right)^{N}=\left[\begin{array}{ll}
m_{11} & m_{12} \\
m_{21} & m_{22}
\end{array}\right]
$$

According to the elements of the above total transfer matrix, transmission and reflection coefficients can be reached as:

$$
\begin{aligned}
t(\omega) & =\frac{2 p_{i}}{\left(p_{F} m_{11}+p_{i} m_{22}\right)-\left(p_{i} p_{F} m_{12}+m_{21}\right)}, \\
r(\omega) & =\frac{\left(m_{11}-m_{22}\right) \cos \theta-\left(m_{12} \cos ^{2} \theta-m_{21}\right)}{\left(m_{11}+m_{22}\right) \cos \theta-\left(m_{12} \cos ^{2} \theta+m_{21}\right)},
\end{aligned}
$$

where the parameters $p_{i}=\left(\sqrt{\varepsilon_{i}} / \sqrt{\mu_{i}}\right) \cos \theta$ and $p_{F}=\left(\sqrt{\varepsilon_{F}} / \sqrt{\mu_{F}}\right) \cos \theta$ also refer to the initial and final media (background medium), in which the incident beam respectively comes from and then enters the medium for TE polarization mode. The parameters will be $p_{i}=\left(\sqrt{\mu_{i}} / \sqrt{\varepsilon_{i}}\right) \cos \theta$ and $p_{F}=\left(\sqrt{\mu_{F}} / \sqrt{\varepsilon_{F}}\right) \cos \theta$ for TM polarization mode. Since the proposed structure is considered to be in a vacuum background medium, $\varepsilon_{i}=\varepsilon_{0}=1$ and $\mu_{i}=\mu_{0}=1$. Subsequently, the total transmittance and reflectance quantities of the structure are defined as:

$$
\begin{gathered}
\mathcal{T}=\frac{p_{F}}{p_{i}}|t(\omega)|^{2}, \\
\mathcal{R}=|r(\omega)|^{2} .
\end{gathered}
$$




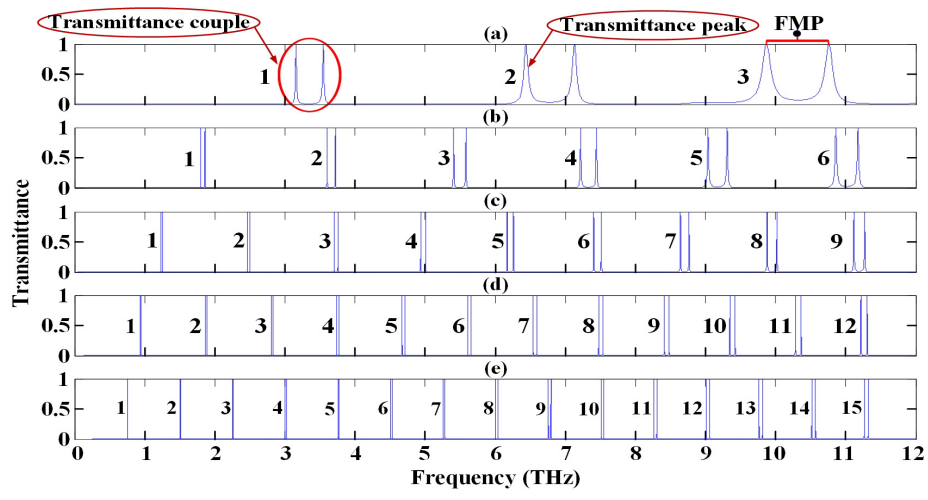

Fig. 1 The transmittance spectrum of the proposed lossless structure versus frequency at $T=4.2 \mathrm{~K}$ and $\theta=0^{\circ}$ with the period number $M=3$ under several values of lattice constants as (a) $d_{A}=10 \mu \mathrm{m}, d_{B}=10 \mathrm{~nm}$, (b) $d_{A}=20 \mu \mathrm{m}, d_{B}=20 \mathrm{~nm}$, (c) $d_{A}=30 \mu \mathrm{m}, d_{B}=30 \mathrm{~nm}$, (d) $d_{A}=40 \mu \mathrm{m}, d_{B}=40 \mathrm{~nm}$ and (e) $d_{A}=50 \mu \mathrm{m}, d_{B}=50 \mathrm{~nm}$ for TE polarization.

\section{Results and discussion}

The arrangement of the proposed structure as an absolute periodic $\mathrm{PC}$ consisting of a low-temperature superconductor layer $(B)$ and a semiconductor layer $(A)$ is $(A B)^{M}$. The transmission spectrum of the structure, and its tunability is studied at $\mathrm{THz}$ spectrum.

The default information in the current research is as follows. The thicknesses of both semiconductor and superconductor layers are respectively considered as $30 \mu \mathrm{m}$ and $30 \mathrm{~nm}$. The critical temperature and London penetration depth of $\mathrm{Nb}$ are respectively $T_{C}=9.2 \mathrm{~K}$ and $\lambda_{0}=83.4 \mathrm{~nm}$. The temperature applied to the structure is considered as $T=4.2 \mathrm{~K}$ (boiling point of liquid $\mathrm{He}$ ). The permittivity of $N b$ and $I n S b$ could be respectively calculated in accordance with equations (1) and (3), and their permeabilities are taken as $\mu_{A}=\mu_{B}=1$.

\subsection{Lattice constants}

In this section, the effect of changing the thickness of the constituent layers has been studied according to the transmittance spectrum diagram. Therefore, the TE polarization type of the transmittance spectrum has been obtained from the different applied lattice constants such as (a) $d_{A}=10 \mu \mathrm{m}, d_{B}=10 \mathrm{~nm}$, (b) $d_{A}=$ $20 \mu \mathrm{m}, d_{B}=20 \mathrm{~nm},(\mathrm{c}) d_{A}=30 \mu \mathrm{m}, d_{B}=30 \mathrm{~nm}$, (d) $d_{A}=40 \mu \mathrm{m}, d_{B}=40 \mathrm{~nm}$ and (e) $d_{A}=50 \mu \mathrm{m}, d_{B}=50 \mathrm{~nm}$ in Fig. 1. The number of periods, and the applied temperature have been respectively considered as $M=3$ and $T=4.2 \mathrm{~K}$, and the angle of incident light is $\theta=0^{\circ}$. The other parameters are also considered as default mentioned above. As clearly shown in Fig. 1, the number of each transmittance couple, the frequency band-gap between the transmittance couple, the band-gap between each transmittance peak in a couple, and the thickness of each peak are tunable via changing the lattice constants. In each step of increasing the lattice constants, three couples of transmittance peaks are added to the transmittance 


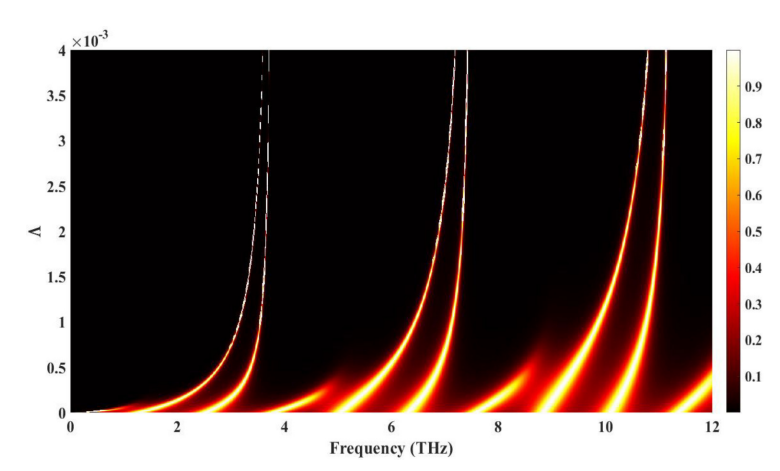

Fig. 2 The transmittance quantity of the proposed lossless structure versus lattice ratio $\Lambda=$ $d_{B} / d_{A}$ and frequency at $T=4.2 K, \theta=0^{\circ}$ and the period number $M=3$ with a constant thickness of $I n S b\left(d_{A}=10 \mu \mathrm{m}\right)$ and the thicknesses range of $\mathrm{Nb}, 0 \mathrm{~nm}$ to $40 \mathrm{~nm}$, for both TE and TM modes.

spectrum, so that there are three couples in (a), six couples in (b), nine couples in (c), twelve couples in (d), and fifteen couples in (e). Therefore, it could be declared that the increase in number of the couples follows the relation $S_{n}=$ $S_{n-1}+3 ; S_{1}=3, n \geq 2$. If the frequency mean point (FMP) between tips of the two peaks in a couple is considered as the frequency position of each couple, it could be observed that the couples are getting closer to each other, as the lattice constants are increasing. In addition, the band-gaps between the couples become somehow wider at a certain lattice constant, as the frequency increases. However, widening of the band-gaps between the couples in terms of frequency is very low at the higher lattice constants, therefore it could be negligible. It could also be understood from Fig. 1 that the band-gaps between the peaks in the couples increase with the increase in the frequency with certain lattice constants. In one hand, the bang-gaps decrease when the lattice constants increase. On the other hand, the band-gaps regularly increases with the increase in the frequency. Similar to the above-mentioned cases, the width of the band-gaps between each transmittance couples is consequently tunable in terms of the lattice constants. As the lattice constants increase, the number of couples and band-gaps becomes greater, and width of the band-gaps regularly decreases. Consequently, the bandgaps also become narrower as the frequency increases under a certain condition of lattice constants. In accordance with Fig. 1, the width of the transmittance peaks is tuned with the lattice constants, therefore the peaks become narrower since the lattice constants increase. Furthermore, they become broader as the frequency increases under a certain condition of lattice constants. The band-gaps between the couples are called the "main transmittance bandgaps", and those between the peaks are called "partial transmittance band-gaps", here.

The transmittance of the proposed structure is also evaluated based on the ratio of the lattice constants. Fig. 2 shows the transmittance versus frequency and lattice ratio $\Lambda=d_{B} / d_{A}$, where $d_{B}=10 \mu \mathrm{m}$, and range of the thickness of layer $A$ considered $10 \mathrm{~nm}$ to $40 \mathrm{~nm}$. As shown in Fig. 2, the peaks shift towards the higher frequencies by a certain value, as $\Lambda$ increases. Furthermore, the width of the peaks and the bandgaps between them also become narrower as $\Lambda$ increases. Both TE 


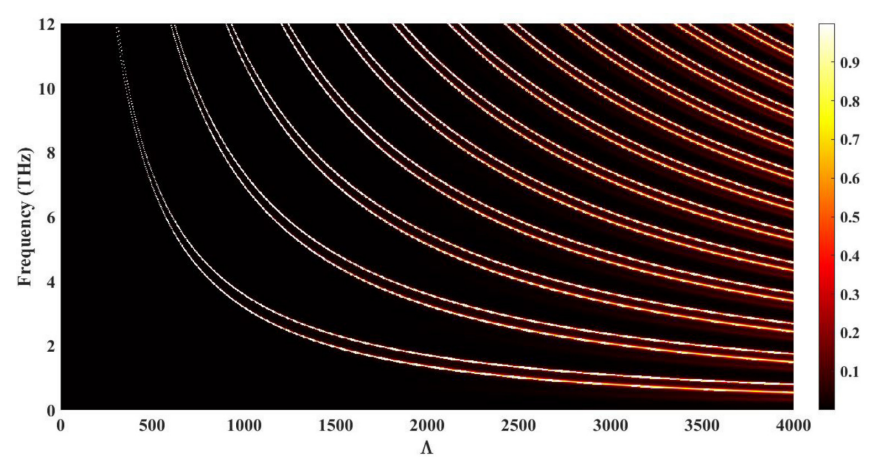

Fig. 3 The transmittance quantity of the proposed lossless structure versus lattice ratio $\Lambda=$ $d_{A} / d_{B}$ and frequency at $T=4.2 \mathrm{~K}, \theta=0^{\circ}$ and the period number $M=3$ with a constant thickness of $\mathrm{Nb}\left(d_{B}=10 \mathrm{~nm}\right)$ and the thicknesses range of $\mathrm{InSb}, 0 \mathrm{~nm}$ to $40 \mu \mathrm{m}$, for both TE and TM modes.

and TM polarization modes of the transmittance quantity are the same as shown in Fig. 2.

On the other hand, the lattice ratio is defined as $\Lambda=d_{A} / d_{B}$, thus the thickness of the superconductor is a fixed value $\left(d_{B}=10 \mathrm{~nm}\right)$, and the range of the lattice constants of the utilized semiconductor is considered as $0 \mu \mathrm{m}$ to $40 \mu \mathrm{m}$. In other words, the consequences of the lattice constant changes relevant to the semiconductor are evaluated on the tunability of the transmittance spectrum, here. As demonstrated in Fig. 3, the transmittance couples shift towards the lower frequencies, as $\Lambda$ increases. By increasing $\Lambda$, the intensity of the peaks is increases, and the band-gaps between the couples become narrower as well. Furthermore, the bandgaps between the peaks become broader and then become somehow narrower, as $\Lambda$ increases from 0 to 4000 in the lower order of the couples (couples which are closer to the axis $\Lambda$ ). However, the bandgaps between the couples almost remain constant in the higher order of the couples. Fig. 3 is the plot of both TE and TM polarized waves.

\subsection{The number of periods}

To study the effects of changes in the period number, the lattice constants are considered as $d_{A}=30 \mu \mathrm{m}$ and $d_{B}=30 \mathrm{~nm}$; the incident angle is $\theta=0^{\circ}$; the temperature applied to the structure is $T=4.2 \mathrm{~K}$ with TE polarization wave at the frequency range of $0 \mathrm{THz}$ to $12 \mathrm{THz}$. According to Fig. 4, the increase in the period number of the proposed structure regularly causes more peaks in the transmittance stacks. Evidently, a transmittance peak is added to the stacks in each step of increasing the period number (from $M=2$ to $M=6$ ). For example, for $M=4$ there are three peaks in each stack, while there are four and five peaks in each stack for $M=5$ and $M=6$, respectively. Therefore, it could be concluded that the number of transmittance peaks follows the relation $S_{n}=S_{n-1}+1 ; n \geq 1$, where $S_{n-1}$ is the stacks number of the transmittance peaks at the previous period number, and $S_{0}=1$ corresponds to the number of the peaks for $M=2$. Fig. 4 shows that the transmittance peaks are too close to each other in a stack at lower 


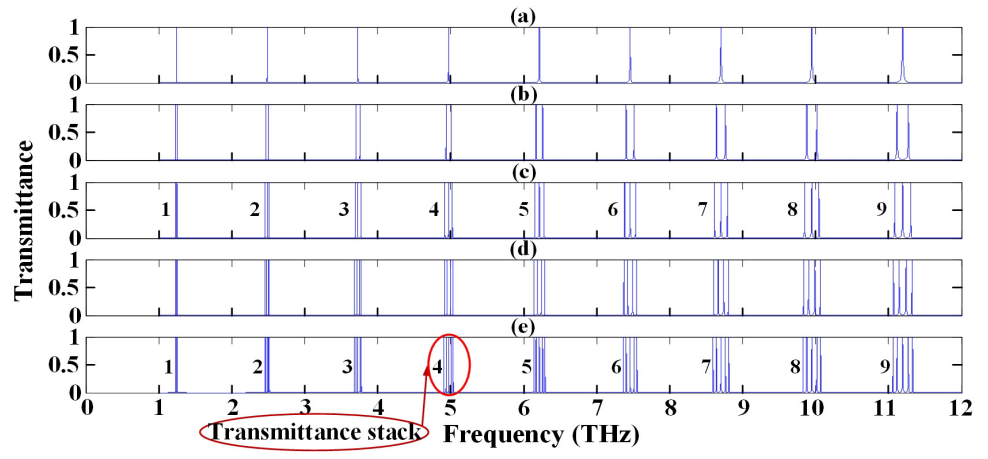

Fig. 4 The transmittance spectrum of the proposed lossless structure versus frequency at $T=4.2 \mathrm{~K}$ and $=0$ with the lattice constants $d_{A}=30 \mathrm{~nm}$ and $d_{B}=30 \mathrm{~nm}$ under several the period numbers $M=$ (a) 2, (b) 3, (c) 4, (d) 5, (e) 6 for TE polarization.

frequencies in certain conditions of the lattice constants and period number, while they are further apart at higher frequencies. Additionally, the transmittance peaks at higher frequencies are somehow broader than the peaks at lower frequencies. As the number of periods increases, the width of the transmittance stacks becomes broader due to the increase in the number of peaks in each step. On the other hand, the main band-gaps between the stacks consequently decrease in each step of increasing $M$. For instance, the width of the bandgap between the third and fourth stack is $1.15 \mathrm{THz}$ for $M=4$ (Fig. 4 (c)), while the width is $1.13 \mathrm{THz}$ for the same stacks at $M=6$ (Fig. $4(\mathrm{e})$ ). This phenomenon also happens in certain conditions of the lattice constants and the period number at higher frequency values. In other words, as the width of the transmittance stacks becomes broader at higher frequencies, the band-gaps between the stacks becomes narrower as well. Generally, the width of the transmittance peaks also becomes wider with increasing the period number.

\subsection{Incidence angles}

Here, PBGs of the transmittance quantity are evaluated with changes of the incidence angle of the $\mathrm{THz}$ beam under several angles such as $\theta=0^{\circ}, 45^{\circ}, 60^{\circ}$, $86^{\circ}$. As shown in Fig. 5, the transmittance spectra and the dispersion quantity of the proposed structure are given at all of the above-mentioned angles for TE modes. It is clear that increasing the incident angles form (a) to (d) causes the peaks and PBGs to be moved towards the higher frequencies. This phenomenon is more tangible for the frequencies greater than $9 \mathrm{THz}$. The shifts of the PBGs at higher frequencies are respectively higher than the those at lower frequencies. Furthermore, the width of the peaks broadens as the frequency increases at a fixed incident angle for TE and TM modes. It could be observed in Fig. 5 that the peaks become narrower when the incident angle increases for TE polarization. Nevertheless, the width of the peaks increases with the increase in the incident angle for TM mode. In accordance with Fig. 6(d), the peaks are so much broadened in the couples, especially at higher frequencies, that they almost merge with each 


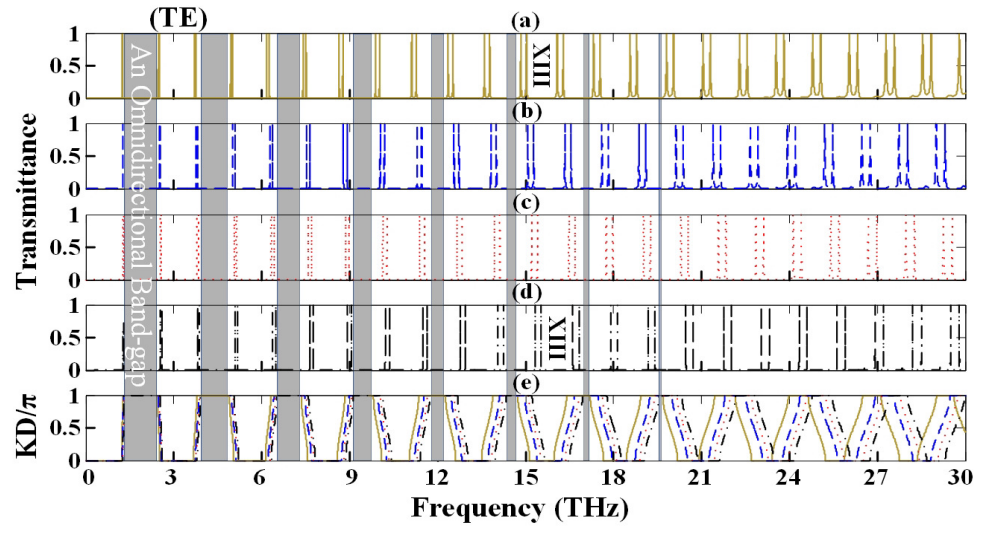

Fig. 5 The transmittance and (e) dispersion $(K D / \pi)$ spectra of the proposed lossless structure versus frequency at $T=4.2 \mathrm{~K}$ and the period number $M=3$ with the lattice constants of $d_{A}=30 \mathrm{~nm}$ and $d_{B}=30 \mathrm{~nm}$ under several incidence angles $\theta=$ (a) $0^{\circ}$, (b) $45^{\circ}$, (c) $60^{\circ}$ and (d) $86^{\circ}$ for TE polarizations.

other. On the other hand, some parts of these PBGs have a maximum dispersion $(K D / \pi=1)$ in some frequency range, and remain invariant across increase in the incident angle from $\theta=0^{\circ}$ to about $\theta=90^{\circ}$. This type of PBGs, called omnidirectional band-gaps, could also stops both TE and TM modes of the incident beam at any angle of incidence. The omnidirectional band-gaps of the proposed structure are shown with gray strips in Fig. 5. It is clear that the width of the omnidirectional band-gaps becomes narrow and narrower, as the frequency increases. The maximum and the minimum width the of the omnidirectional band-gaps are the same and respectively equal to $\Delta f_{\text {omni,max }}=1.144 \mathrm{THz}$ and $\Delta f_{\text {omni,min }}=0.13$ $\mathrm{THz}$ for TE and TM modes of the transmittance spectra.

\subsection{Temperature}

In accordance with relations (3) and (7), permittivity of the utilized superconductor depends on temperature. Therefore, values of the permittivity and consequently values of the transmittance could be tuned with the temperature. For evaluating the effects of changing the temperature on tuning the transmittance spectrum, several external temperatures are applied to the structure, $T=1 \mathrm{~K}, 4.6 \mathrm{~K}, 6.5 \mathrm{~K}$, $9.1 K, 9.2 K$ and $9.4 K$, whose corresponding transmittance spectra are given in Fig. 6. As could be observed, the applied temperatures contain the under critical, critical $\left(T_{C}\right)$ and greater than critical temperatures. In accordance with the figure, it could be stated that the increase in the temperature from $1 K$ (a) to $6.2 K$ (c) somehow results in the broadening of the peaks and the partial band-gaps in the couples. For instance, consider couple 9 in Fig. 6(a), (b) and (c). It is undeniable that the amount of the tunability is too slight from $1 K$ (a) to $\frac{1}{2} T_{C}$ (b), thus it could be ignored. As shown in the figure, when the temperature tends to $T_{C}$ (Fig. 6(d)), the filtering property weakens, and does not satisfy the expectation that we had in the previous sections. On the other hand, the transmittance of the 


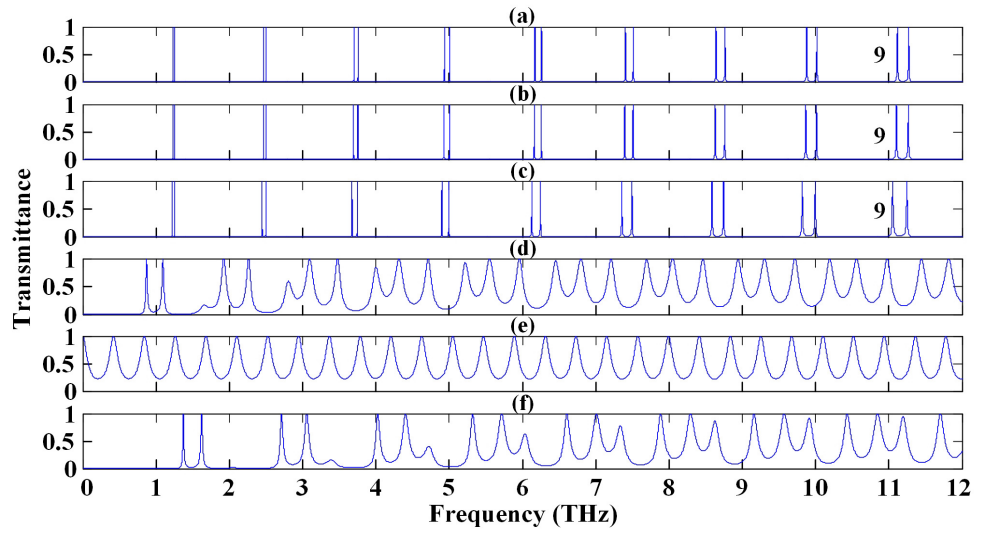

Fig. 6 The transmittance spectrum of the proposed lossless structure versus frequency at $\theta=0^{\circ}$ with the periodic number $M=3$ and the lattice constants $d_{A}=30 \mathrm{~nm}$ and $d_{B}=30 \mathrm{~nm}$, under several applied external temperature below, at and greater than the critical temperature of the utilized superconductor $(N b)$ as $T=$ (a) $1 K$, (b) $4.6 K$, (c) $6.5 K$, (d) $9.1 K$, (e) $9.2 K$, and (f) $9.4 K$ for TE polarization.

structure does not follow the rules of the tunability, which exist for $T<\frac{1}{2} T_{C}$. If the applied external temperature reaches $T_{C}$, the minimum values of the bandgaps are not zero, thus the smallest transmittance value of the band-gaps is about 0.22. The applied temperatures greater than $T_{C}$ (Fig. 6(f)), which cause layer $B$ not to be a superconductor anymore, create an electric resistivity change of 0.688 $\Omega m$ for the frequency range of $0 \mathrm{THz}$ to $12 \mathrm{THz}$, in accordance with equation (6). Here, the number of peaks decreases compared to (d) and (e), and there is no band-gap with zero-transmittance for the frequencies greater than $\sim 4 \mathrm{THz}$. When the temperature tends to $T_{C}$ and greater than $T_{C}$, the transmittance peaks become broader and somehow disorganized in such an undesirable way, and their behavior becomes irregular, so that the filtering phenomenon does not properly work, anymore.

\subsection{Central defect layer}

In accordance with Fig. 7, the proposed structure is also evaluated with a central defect layer. In this section, a vacuum layer $\left(\varepsilon_{V}=1\right.$ and $\left.\mu_{V}=1\right)$ is considered as the low-permittivity-value defect layer, and another dielectric layer with $\varepsilon_{D}=10$ and $\mu_{D}=1$, which could be a type of glass, is considered as the high-permittivityvalue defect layer. Although, the permittivity of the air defect layer is close to 1 , and it is less expensive and more accessible than vacuum, utilizing such layer at $T=4.2 \mathrm{~K}$ is pretty difficult, thus replacing a central vacuum defect layer is comparatively more convenient. The transmittance spectrum of each structure relevant to the deposition of the vacuum and the dielectric layer is also studied for several thicknesses of the central defect layers. Fig. 8 which corresponds to Fig. 7(a), demonstrates the transmittance spectrum of the structure with several orders of the thickness of the defect layer as $d_{V}=40 \mathrm{~nm}, 80 \mathrm{~nm}, 40 \mu \mathrm{m}$ and $80 \mu \mathrm{m}$. 


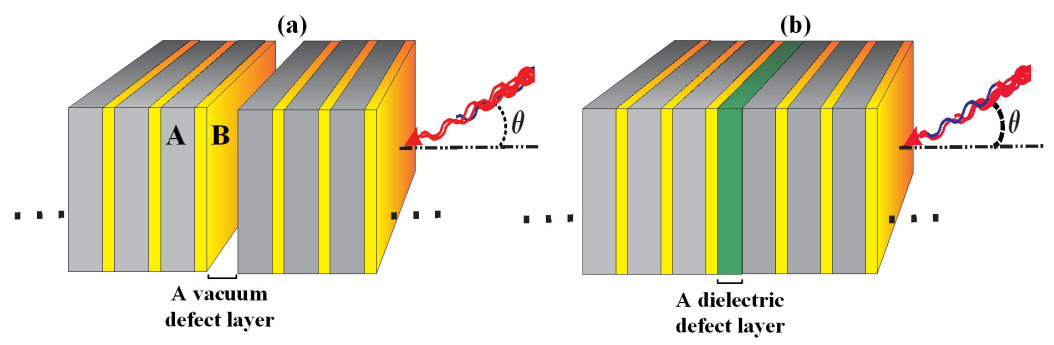

Fig. 7 The schematics of the proposed structures containing a micro semiconductor and a nano superconductor layer with (a) a central vacuum defect layer and (b) a central dielectric defect layer.

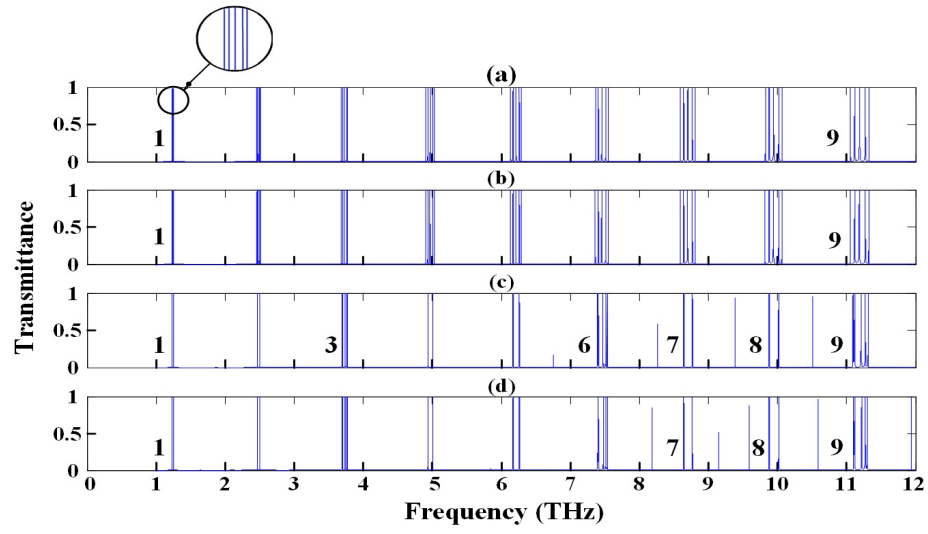

Fig. 8 The transmittance spectrum of the proposed lossless structure containing a central vacuum defect layer $\left(\varepsilon_{V}=1\right.$ and $\left.\mu_{V}=1\right)$, under several orders of thickness $d_{V}=($ a) $40 \mathrm{~nm}$, (b) $80 \mathrm{~nm}$, (c) $40 \mu \mathrm{m}$ and (d) $80 \mu \mathrm{m}$, at $T=4.2 \mathrm{~K}$ and $\theta=0^{\circ}$ with the lattice constants $d_{A}=30 \mathrm{~nm}, d_{B}=30 \mathrm{~nm}$, and the period number of $M=3$ for TE polarization.

As it is obvious, both nano and micro scales of the central defect layers are applied to the structure. Both of the proposed constructions relevant to schematics (a) and (b) in Fig. 7 are considered as $(A B)^{M} C(A B)^{M}$, where the period number is $M=3$, the incidence angle is equal to $\theta=0^{\circ}$, and the lattice constants are $d_{A}=30 \mu \mathrm{m}$ and $d_{B}=30 \mathrm{~nm}$. As illustrated in Fig. 8(a) and 8(b), nine transmittance stacks emerge, so that there are five peaks in each stack. Since the peaks are too close to each other at lower frequencies, they seem to coincide with each other. The magnified part of Fig. 8(a) clearly demonstrates the dispersion of the peaks in stack 1 . In accordance with the numerical data, the width and the frequency position of the stacks remain invariant with increase in the thickness of the vacuum defect layer at the nanoscale. Therefore, the presence of the central defect layer gives us a desirable multichannel $\mathrm{THz}$ filter, and changing $d_{V}$ in the scale of nanometer $(0 \mathrm{~nm}$ to $100 \mathrm{~nm})$ has no effect on tuning the transmittance spectrum. The width of stacks 1 and 9 are respectively $\Delta f_{1, V}=0.033 \mathrm{THz}$ and $\Delta f_{9, V}=0.28 \mathrm{THz}$, and their FMPs are also $f_{1, V}=1.24 \mathrm{THz}$ and $f_{9, V}=11.09 \mathrm{THz}$, respectively. Accordingly, the width of stack 9 is 8.48 times broader than stack 1 here. If the vacuum defect 


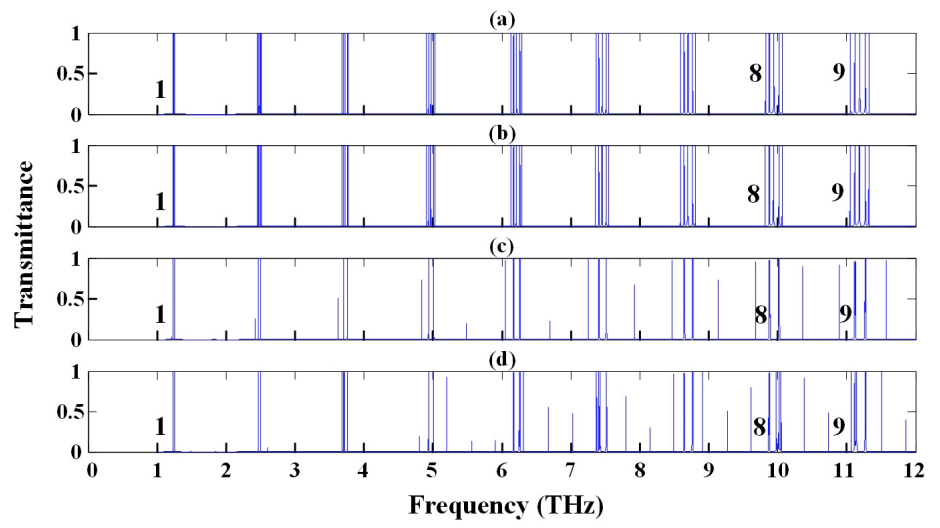

Fig. 9 The transmittance spectrum of the proposed lossless structure containing a central dielectric defect layer $\left(\varepsilon_{D}=10\right.$ and $\left.\mu_{D}=1\right)$, under several orders of thickness $d_{V}=(\mathrm{a})$ $40 \mathrm{~nm}$, (b) $80 \mathrm{~nm}$, (c) $40 \mu \mathrm{m}$ and (d) $80 \mu \mathrm{m}$ at $T=4.2 \mathrm{~K}$ and $\theta=0^{\circ}$ with the lattice constants $d_{A}=30 \mathrm{~nm}, d_{B}=30 \mathrm{~nm}$, and the period number of $M=3$ for TE polarization.

layer becomes broader as much as the microorder, the transmittance spectra will be significantly changed. As shown in Fig. 8(c), some of the transmittance stacks have two peaks and other stacks have five peaks. It is obvious that the five-peak stacks are repeated twice in between (stacks 3,6 and 9). As obviously observable in spectrum (c), several narrow peaks are emerged due to the presence of the central vacuum defect layer in the PBG areas at the higher frequencies $(8 \mathrm{THz}$ to $11 \mathrm{THz}$ ) like the defect mode between stacks 7 and 8. The transmittance spectrum corresponding to $d_{D}=80 \mu \mathrm{m}$ (d) is almost the same as spectrum (c). But, the number and the transmittance values of the defect modes are greater. For instance, there are two defect modes between stacks 7 and 8 .

In accordance with Fig. 9, spectra (a) and (b) are exactly the same as spectra (a) and (b) in Fig. 8. This means, there is no difference between the transmittance spectra corresponding to the structures with high- and low-permittivity central nano defect layer. In accordance with Fig. 8(a), 8(b), 9(a), 9(b), and 4(e), the transmittance spectra with the central nano defect layers at $M=3$, are the same as the spectrum that corresponds to the structure without central defect layer at $M=6$. As obviously shown in Fig. 9(c) and $9(\mathrm{~d})$, there are two or three peaks in each stack, which become further apart in the stacks with increasing frequency. Here in Fig. 9(d), the number of defect modes are higher than the modes in Fig. 9(c). Accordingly, the number of defect modes in Fig. 9(d) are higher than the modes in Fig. 8(c) and 10(d).

\section{Conclusion}

In accordance with the results of the current study, it is found that using a constituent element of superconductor could make the structure well-behaved, optically. The deposition of a nano superconductor defect layer of $\mathrm{Nb}$ caused the structure to yield high tunable transmittance peaks and PBGs. On the other 
hand, InSb at $T=4.2 \mathrm{~K}$, which approximately had the permittivity value of 15.68 , caused the structure to result in the achieved transmittance spectra. One of the considerable phenomena relevant to the transmittance was omnidirectional PBGs. Although this type of PBGs could also happen for the other PC structures, the amount of omnidirectional property and their tunability were significant. Fig. 2 and 3 illustrate that the ratio of the lattice constants was chosen appropriately in this study. They also show that the ratio plays a considerable role in the tunability and intensity of the transmittance spectrum. Using a relatively low- and high-permittivity central defect layer with different thicknesses in the structure revealed that the central nano-scale defect layer resulted in a well-organized, and high regular transmittance peaks and stacks. On the other hand, the micro-scale defect layer emerged several tunable defect modes in the PBG areas at higher frequencies. Moreover, there was an acceptable percent of the transmittance in presence of the damping coefficient in the frequency range of $5 \mathrm{THz}$ to $12 \mathrm{THz}$, so that the percentage was equal to $40 \%$ at $f=5 \mathrm{THz}$, and it reached $80 \%$ at $f=11.27 \mathrm{THz}$. The utilized superconductor and semiconductor, which resulted in such well-behaved multi-channel transmittance peaks, are the main differences between the current study and [32]. Here, the desirable frequencies could be selectively chosen to be transmitted by the tunability of the structure. Additionally, the proposed structure in [33] consists of a high-temperature superconductor and $G a A s$, while we had a low-temperature superconductor and InSb, in which the tunability and speciality of the current structure were comparatively studied at a higher level. The present position, future prospect, and advancing of the $\mathrm{THz}$ technology have been a focal point, and the proposed structure could be used in modern $\mathrm{THz}$ communications, $\mathrm{THz}$ selective multi-channel frequency filters, integrated circuits, smart windows, low-temperature sensors, etc.

\section{Funding}

No funds, grants, or other support was received.

\section{Conflicts of interest}

The authors have no conflicts of interest to declare that are relevant to the content of this article.

\section{Data availability}

All data generated or analysed during this study are included in this published article

\section{References}

1. E. Bründermann, H.W. Hübers, M.F. Kimmitt, Terahertz techniques, vol. 151 (Springer, 2012) 
2. S. Romanenko, R. Begley, A.R. Harvey, L. Hool, V.P. Wallace, Journal of The Royal Society Interface 14(137), 20170585 (2017)

3. D. Huang, Technology Review $107(1), 3250$ (2004)

4. Y.B. Ji, J.M. Kim, Y.H. Lee, Y. Choi, Y.M. Huh, S.J. Oh, Y.W. Koh, J.S. Suh, et al., Journal of Infrared, Millimeter, and Terahertz Waves 40(2), 247 (2019)

5. M. Mernea, O. Calborean, O. Grigore, T. Dascalu, D.F. Mihailescu, Optical and Quantum Electronics 46(4), 505 (2014)

6. V. Vaks, A. Semenova, Y.S. Guseva, A. Panin, Optical and Quantum Electronics 49(5), $193(2017)$

7. İ.O. Yıldırım, V.A. Özkan, F. İdikut, T. Takan, A.B. Şahin, H. Altan, Optical and Quantum Electronics 48(7), 1 (2016)

8. C. Cao, Z. Zhang, X. Zhao, T. Zhang, Optical and Quantum Electronics 52(4), 1 (2020)

9. B. Qin, Z. Li, Z. Luo, Y. Li, H. Zhang, Optical and Quantum Electronics 49(7), $1(2017)$

10. C. Zhang, T. Ning, J. Li, L. Pei, H. Chen, Optical and Quantum Electronics 47(8), 2717 (2015)

11. P. Bawuah, D. Markl, D. Farrell, M. Evans, A. Portieri, A. Anderson, D. Goodwin, R. Lucas, J.A. Zeitler, Journal of Infrared, Millimeter, and Terahertz Waves pp. 1-20 (2020)

12. H. Hoshina, Y. Saito, T. Furuhashi, T. Shimazaki, M. Sawada, Y. Hioki, C. Otani, Journal of Infrared, Millimeter, and Terahertz Waves 41(3), 265 (2020)

13. Y. Yu, Z. Zhang, T. Zhang, X. Zhao, Y. Chen, C. Cao, Y. Li, K. Xu, Optical and Quantum Electronics 52(5), 1 (2020)

14. K. Fukunaga, Thz technology applied to cultural heritage in practice (Springer, 2016)

15. S. Sahu, J. Ali, P.P. Yupapin, G. Singh, K. Grattan, Optical and Quantum Electronics $\mathbf{5 0}(8), 1$ (2018)

16. Z. Yang, K. Chen, C.g. Wang, X. She, N. Li, X.w. Shu, Optical and Quantum Electronics $\mathbf{5 2}(2), 1(2020)$

17. W. Liu, H. Ma, A. Walsh, Renewable and Sustainable Energy Reviews 116, 109436 (2019)

18. A. Kumar, P. Singh, K.B. Thapa, Optical and Quantum Electronics 52(10), 1 (2020)

19. K. Okamoto, K. Tsuruda, S. Diebold, S. Hisatake, M. Fujita, T. Nagatsuma, Journal of Infrared, Millimeter, and Terahertz Waves 38(9), 1085 (2017)

20. S. Azizi, S. Nouri-Novin, M.M. Seyedsharbaty, F.B. Zarrabi, Optical and Quantum Electronics 50(6), 1 (2018)

21. X. Yu, R. Yamada, J.Y. Kim, M. Fujita, T. Nagatsuma, in 2018 Progress in Electromagnetics Research Symposium (PIERS-Toyama) (IEEE, 2018), pp. 599-605

22. J. Liu, M. Yao, L. Shen, Journal of Materials Chemistry C 7(11), 3121 (2019)

23. A. Baseri, A. Keshavarz, A. Hatef, Journal of Applied Physics 127(21), 214304 (2020)

24. K. Sreejith, N.M. Dsouza, V. Mathew, Physica C: Superconductivity and its Applications 540, 44 (2017)

25. W. Withayachumnankul, B.M. Fischer, D. Abbott, Optics Communications 281(9), 2374 (2008)

26. F. Qiao, C. Zhang, J. Wan, J. Zi, Applied Physics Letters 77(23), 3698 (2000)

27. D. Wu, N. Fang, C. Sun, X. Zhang, W.J. Padilla, D.N. Basov, D.R. Smith, S. Schultz, Applied Physics Letters 83(1), 201 (2003)

28. T.D. Drysdale, R.J. Blaikie, D.R. Cumming, Applied physics letters 83(26), 5362 (2003)

29. H. Němec, L. Duvillaret, F. Garet, P. Kužel, P. Xavier, J. Richard, D. Rauly, Journal of applied physics $\mathbf{9 6}(8), 4072$ (2004)

30. H.C. Hung, C.J. Wu, S.J. Chang, Journal of Applied Physics 110(9), 093110 (2011)

31. S.K. Srivastava, Journal of Superconductivity and Novel Magnetism 27(1), 101 (2014)

32. A.H. Aly, W. Sabra, Journal of Superconductivity and Novel Magnetism 29(8), 1981 (2016)

33. F. Segovia-Chaves, H. Vinck-Posada, Physica C: Superconductivity and its Applications 553, 1 (2018)

34. Y. Trabelsi, Optical and Quantum Electronics 53(2), 1 (2021)

35. A.B. Asl, A. Rostami, I. Amiri, Optical and Quantum Electronics 52(3), 1 (2020)

36. W. Belhadj, A.N. Al-Ahmadi, Optical and Quantum Electronics 53(1), 1 (2021)

37. J. Cos, J. Ferré-Borrull, J. Pallarès, L. Marsal, Optical and quantum electronics 42(8), 487 (2011)

38. S. Wang, L. Kang, D.H. Werner, Scientific reports 8(1), 1 (2018)

39. S. Howells, L. Schlie, Applied Physics Letters 69(4), 550 (1996)

40. M. Tinkham, Introduction to superconductivity (Courier Corporation, 2004)

41. F. Segovia-Chaves, H. Vinck-Posada, Physica C: Superconductivity and its Applications 553, $1(2018)$

42. D. Halliday, R. Resnick, J. Walker, Fundamentals of physics (John Wiley \& Sons, 2013) 
Figures

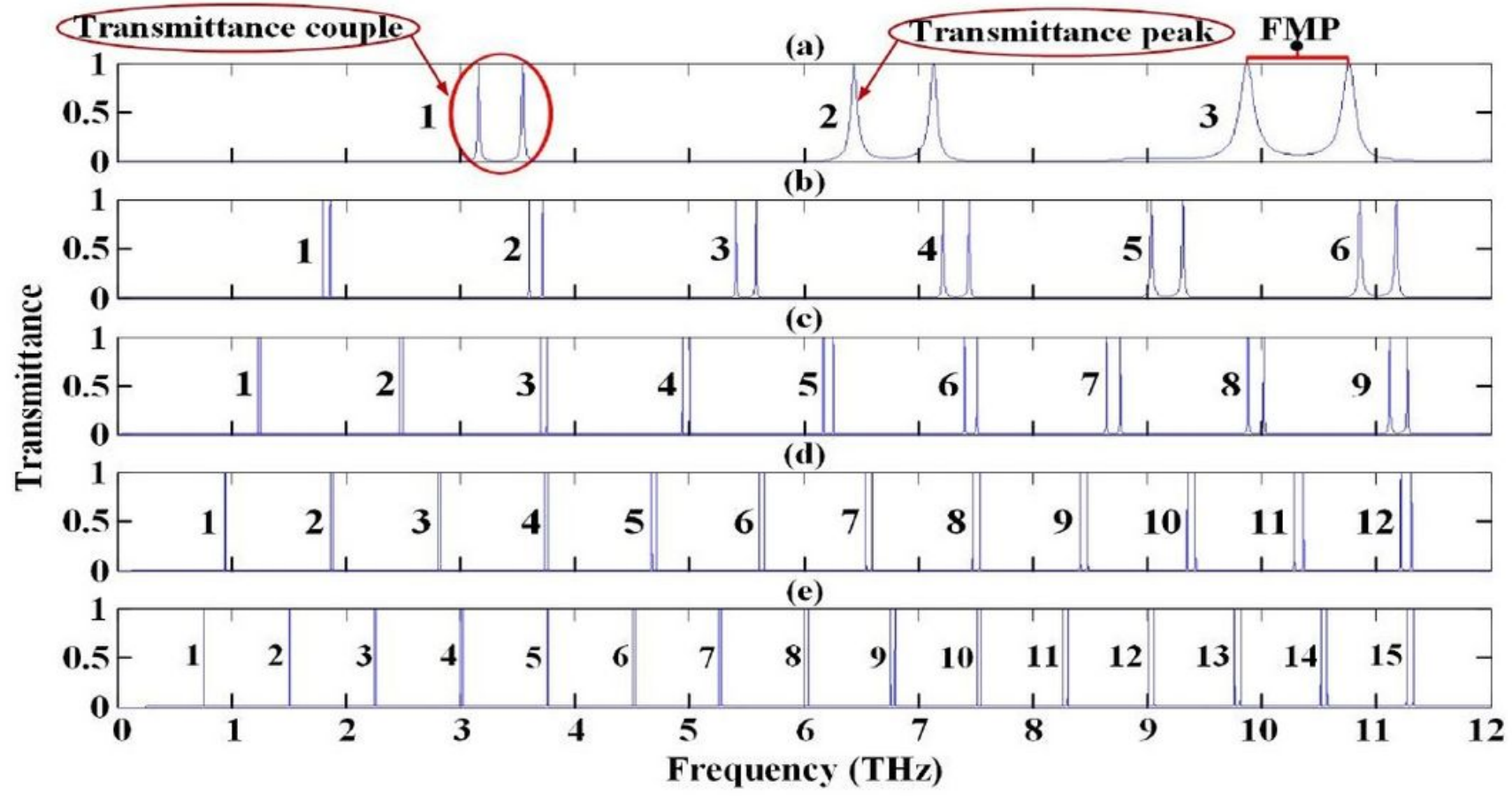

Figure 1

Please see the Manuscript PDF file for the complete figure caption

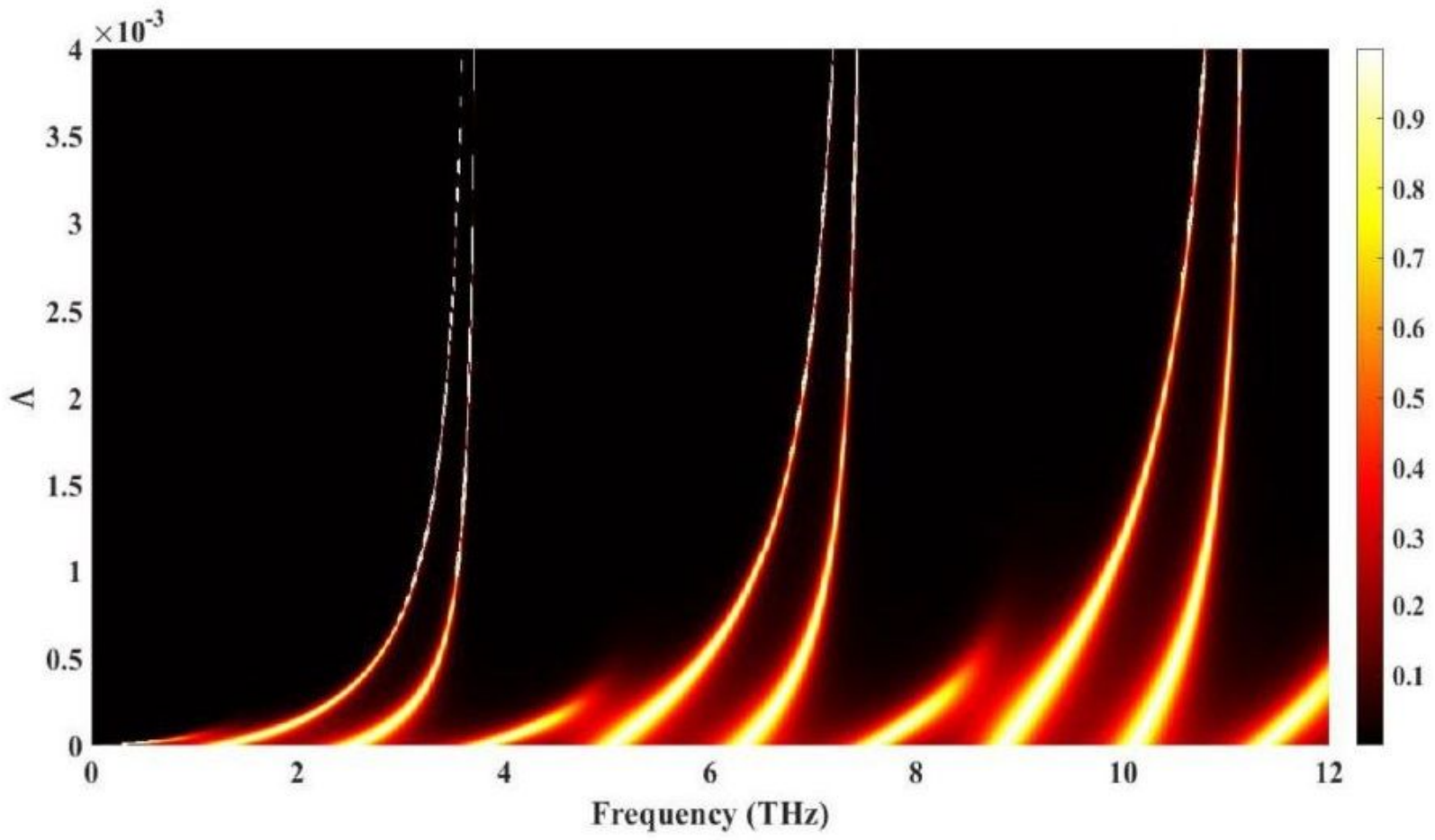

Figure 2 
Please see the Manuscript PDF file for the complete figure caption

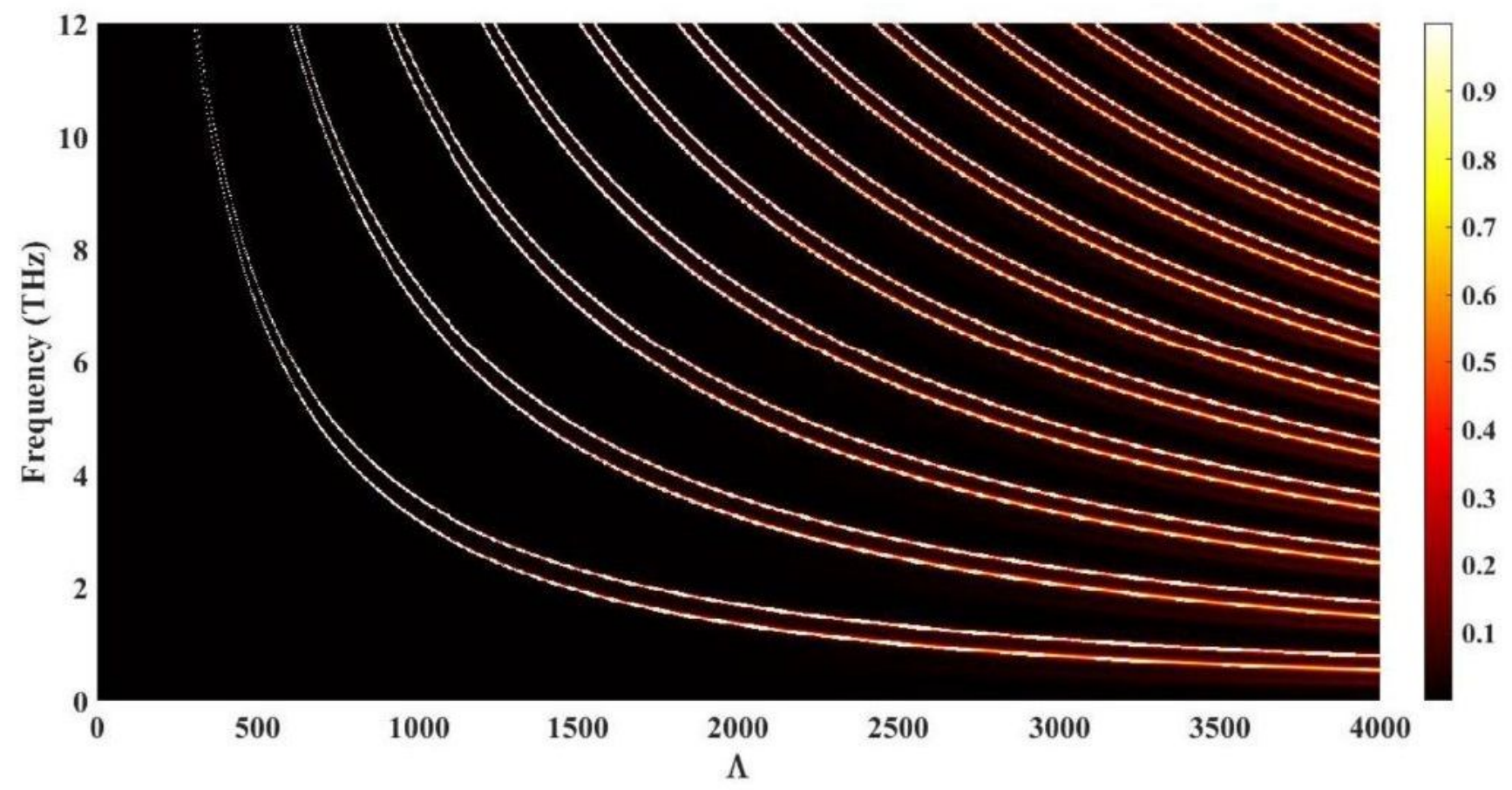

Figure 3

Please see the Manuscript PDF file for the complete figure caption

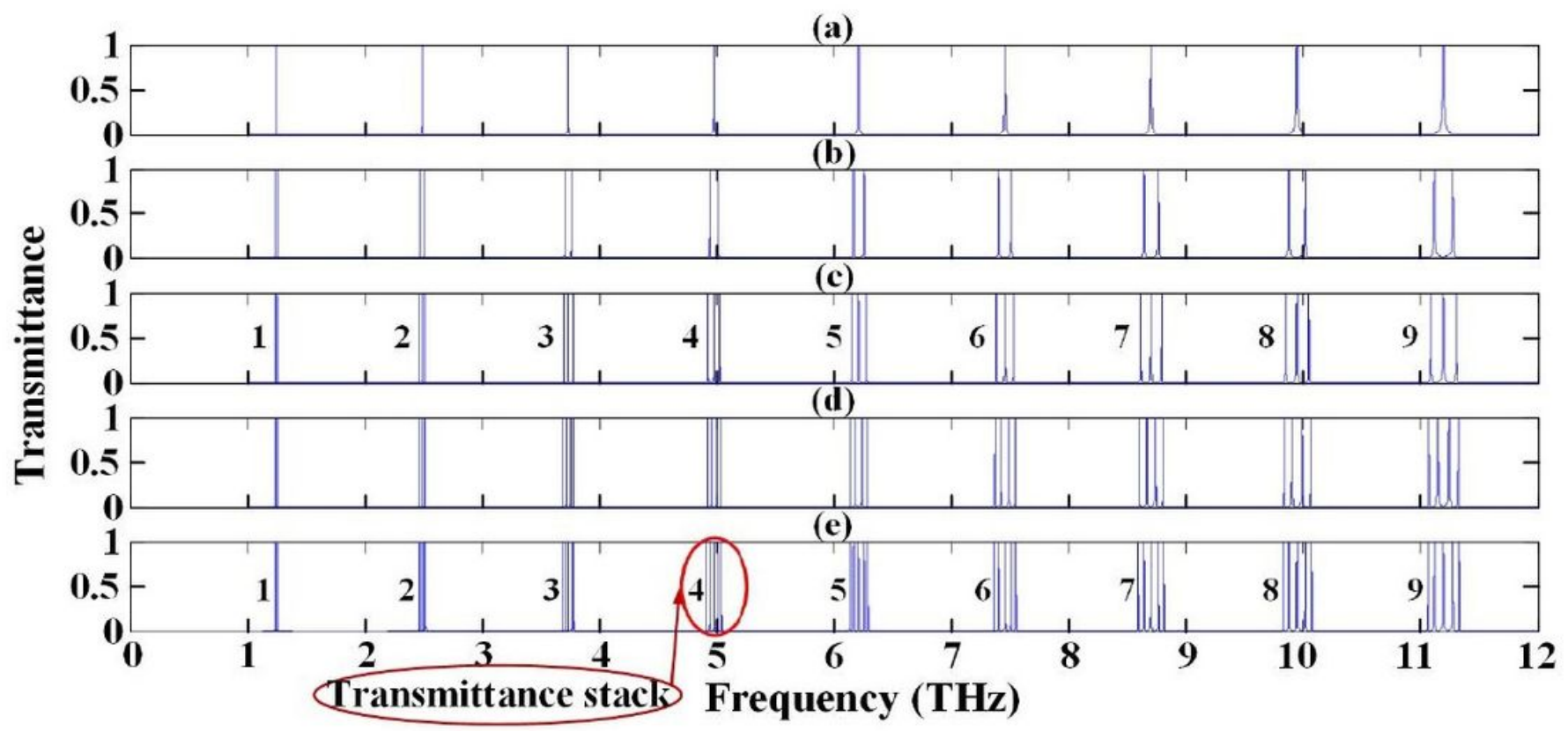

Figure 4

Please see the Manuscript PDF file for the complete figure caption 




Figure 5

Please see the Manuscript PDF file for the complete figure caption

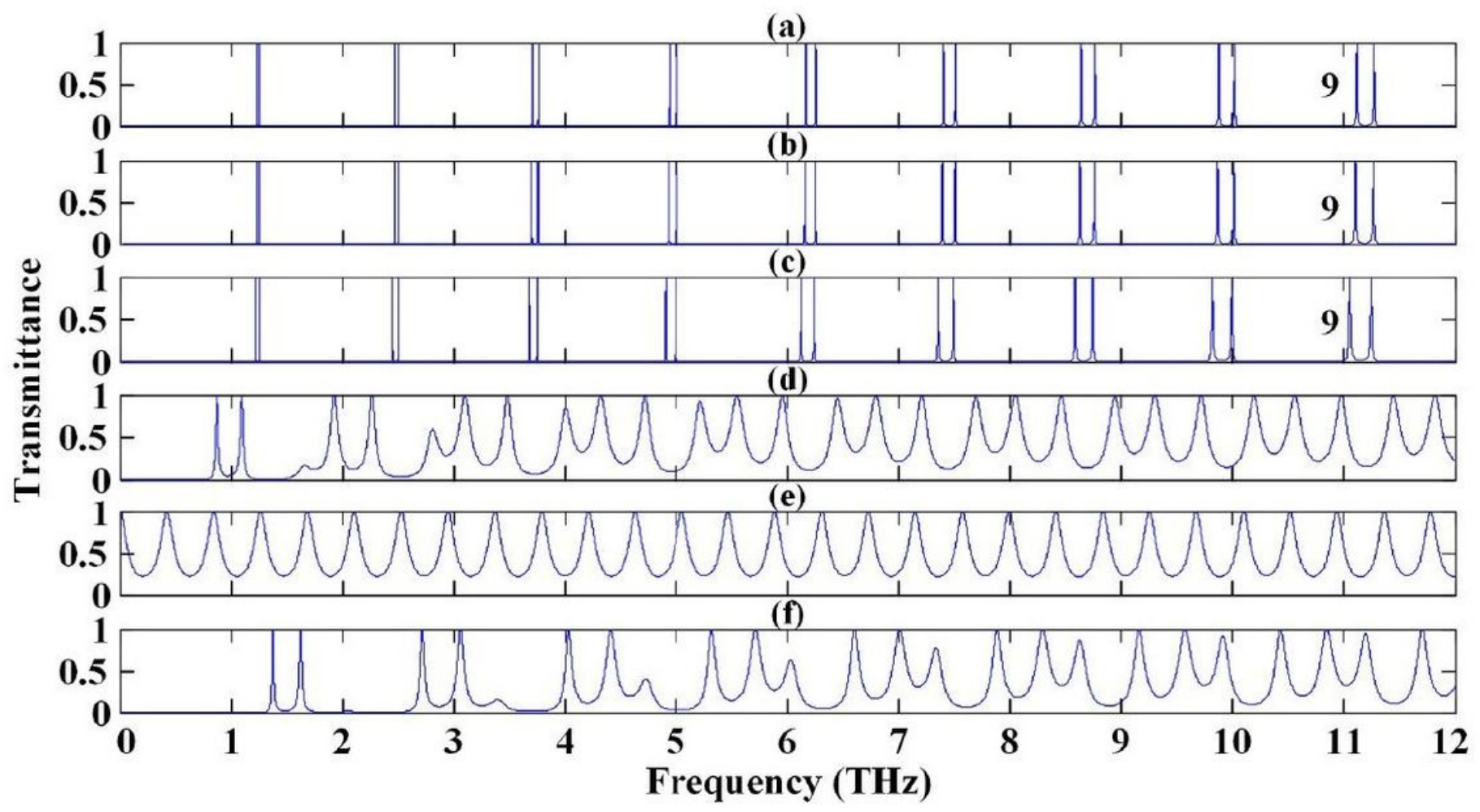

Figure 6 
Please see the Manuscript PDF file for the complete figure caption

(a)

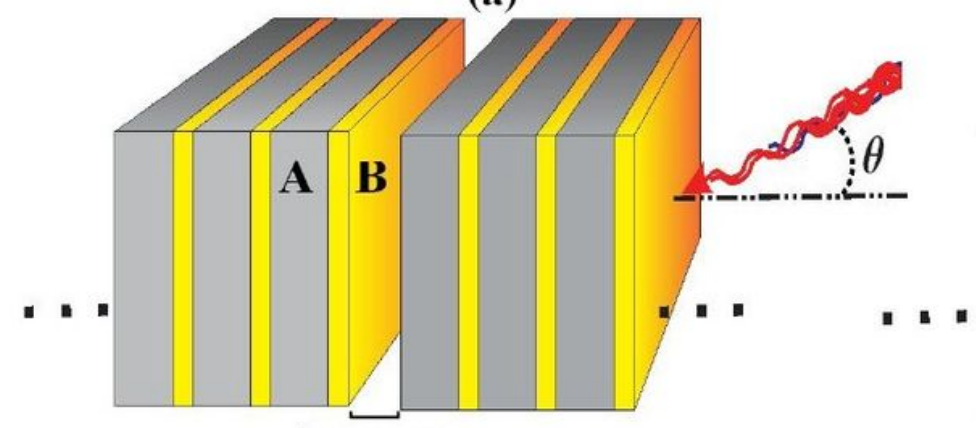

A vacuum

defect layer (b)

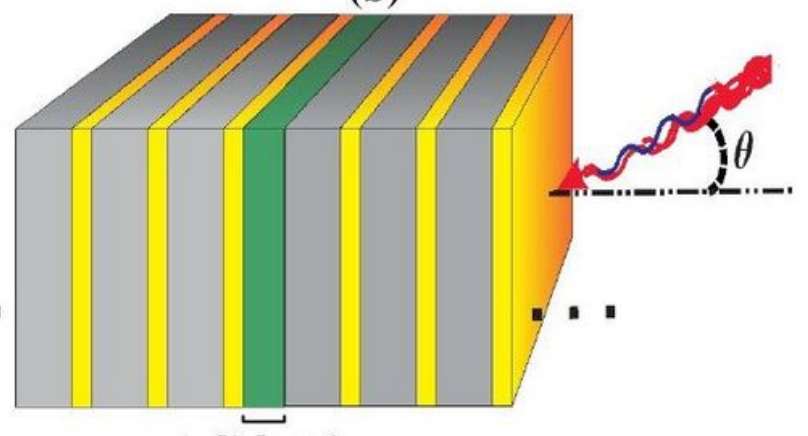

A dielectric defect layer

Figure 7

Please see the Manuscript PDF file for the complete figure caption

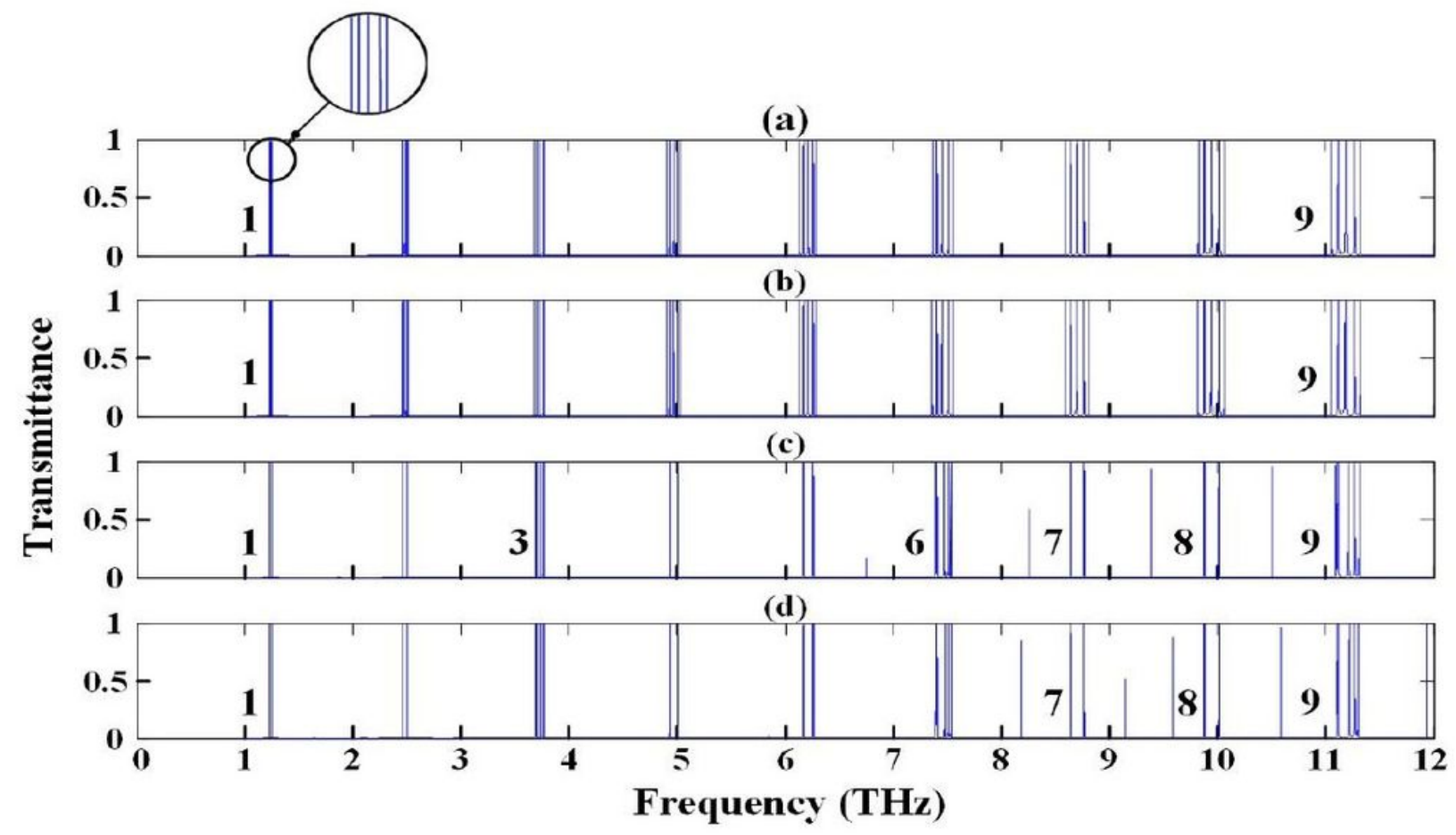

Figure 8

Please see the Manuscript PDF file for the complete figure caption 
(a)

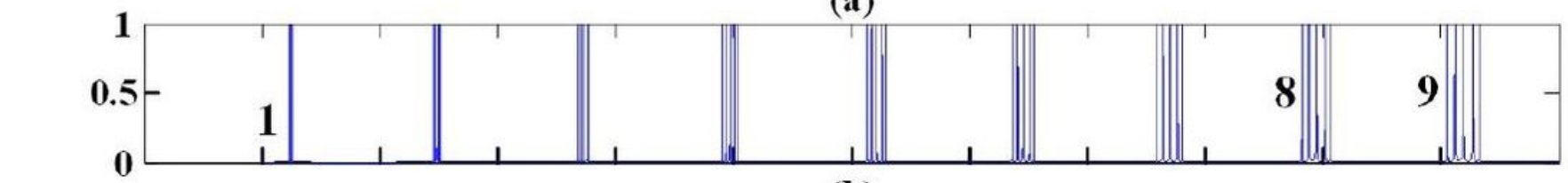

(b)
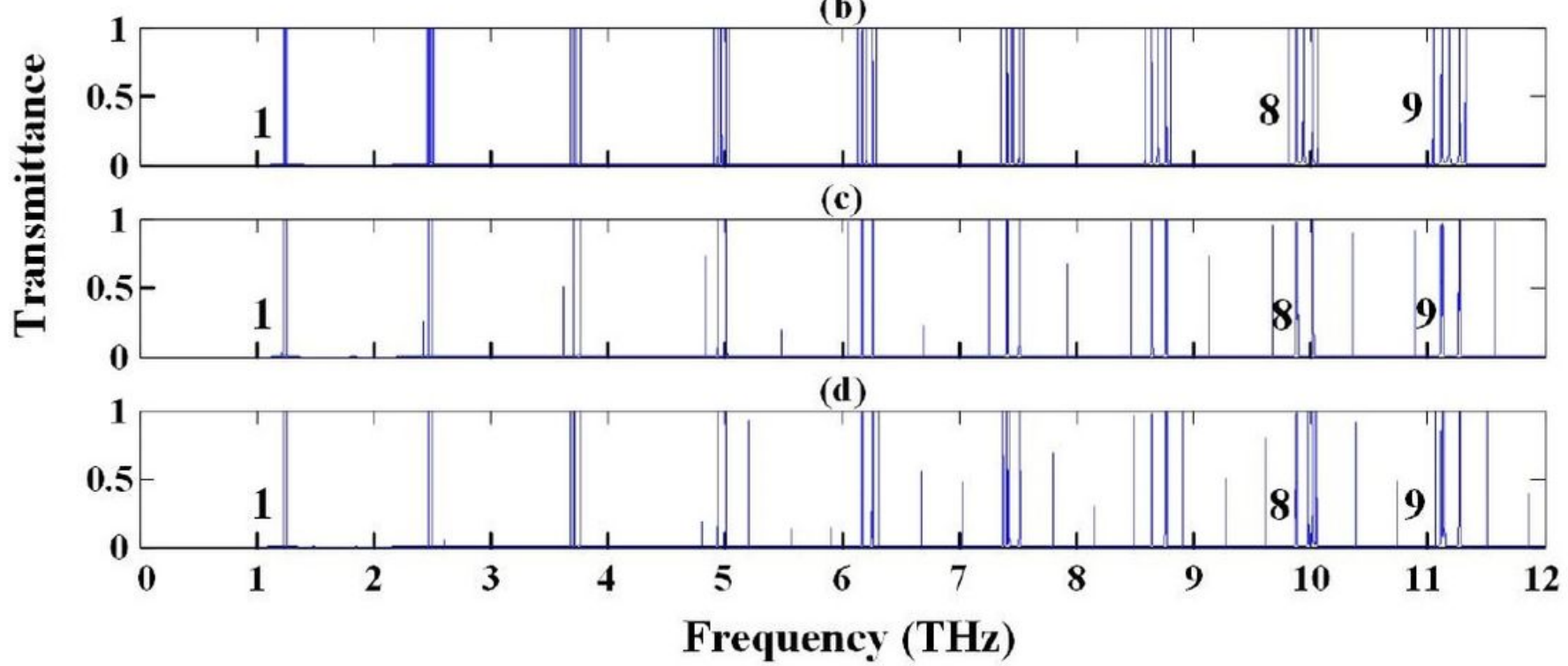

Figure 9

Please see the Manuscript PDF file for the complete figure caption 